\title{
Telemetry System at Ground Control Station of Atmospheric Balloon Payload Using Delphi 7
}

\author{
Helfy Susilawati ${ }^{1}$, Akhmad Fauzi Ikhsan ${ }^{2}$, Mugits Rabbani ${ }^{3}$, Ade Rukmana ${ }^{4}$, Teddy Mulyadi \\ Hidayat $^{5}$ \\ \{helfy.susilawati@uniga.ac.id ${ }^{1}$,txfauzi@gmail.com²,mugitrade@gmail.com³ \\ ade77rukmana@gmail.com ${ }^{4}$,teddy.mulyadi@uniga.ac.id ${ }^{5}$ \}
}

Universitas Garut ${ }^{1}$, Universitas Garut ${ }^{2}$, Universitas Garut ${ }^{3}$, Universitas Garut ${ }^{4}$, Universitas Garut ${ }^{5}$

\begin{abstract}
Telemetry is a communication process by which an object measurement is sent to a remote point through a wired or wireless data transmission process. This study designs a telemetry system using Delphi 7 , which is used to display data parameter from an atmospheric balloon payload. Information transmission from the payload to the computer is facilitated by $433 \mathrm{MHz}$ radio modules. Information from the payload is processed and displayed on the application interface in the form of numerical and graphical data. These data are saved as a *txt file. The interface is designed using $96008 \mathrm{~N} 1$ (9600 baud, 8 data bits, no parity, and 1 stop bit) serial protocols. The displayed parameters include air temperature, air pressure, air humidity, altitude, transverse lines and longitudinal lines. The data history can be read by notepad or other similar Windows applications.
\end{abstract}

Keywords: Telemetry, $433 \mathrm{MHz}$ radio, Delphi 7

\section{Introduction}

A payload is the substance carried by a sonde balloon or a radiosonde into the atmosphere about $10 \mathrm{~km}$ above sea level. The radiosonde works at a frequency of $433 \mathrm{MHz}$. It measures atmospheric parameters and monitors conditions above the maritime area and transmits data to the Ground Control Station (GCS) [1]. The payload consists of one or more instruments including an electronic control system and software [2]. Radio telemetery is frequently used for its mobility and applicability in turbulent locations with entrapped air, such as hydroelectric dam tailraces [3]. A telemetery technology measures a parameter of an object. The measurements are transmitted to receiving equipment through a wired and wireless network [4]. The data transmission is usually one-way, from the sensor to the data logger system [5]. It is necessary to ensure error-free data transmission and reproduction [6]. Therefore, data transmission from the payload to GCS requires software that serves as a data receiver, data reader, and data processor. The software interface displays data changes in the 3DR $433 \mathrm{MHz}$ radio (ground). The software also connects the antenna tracker to personal computer at the ground segment (GS).

The telemetry system has been previously used underwater to study fish behavior [7] and in medical-surgical units for monitoring purposes [8]. Telemetry is frequently used in the monitoring of complicated medical patients without rigorous evidence supporting its use.[9] For another case, most of the research on modern healthcare systems, especially in health monitoring, is suggested for long term monitoring of patients to collect health status information or localization and 
tracking information. [10] In this study, the telemetry system is used to monitor air temperature, pressure, humidity at a particular latitude and latitude in a particular time. Such information is sent a payload to the monitoring device interface which will be displayed using Delphi 7 in the form of numerical and graphical data and stored a *txt (tab separated value) file format.

\section{Method}

This study was conducted using a prototyping. Prototyping is one method of exploring, both individually and collaboratively, a solution space (mathematical entity) for a problem [11] Prototyping method through a procedure consisting of planning, specification, design, and results [12]. The prototype-based model is specially characterized by one idea, the object is the only abstraction provided to the programmer [13]. A prototype is an enactable mock-up or model of a software system that enables evaluation of features or functions through user and developer interaction with operational scenarios. [14] But, for the software, prototype has a bit different. The basic activities or phases to be performed for developing a software system are Determination of System es Requirements, Design of system, Development (coding) of software, and System Testing [15] Data obtained from the radio is displayed on a PC. The output data is sent to Arduino Tracker, which also is shown in the display as illustrated in Figure 1.

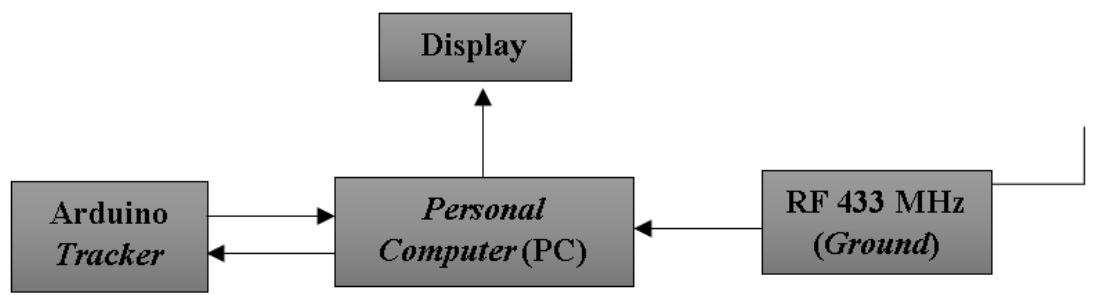

Figure 1. PC Block Diagram with Tracker

Figure 1 shows that data will be sent by a payload to a PC. An interface is required to read the received data. The $\mathrm{PC}$ will send the data to Arduino tracker, and in its turn, Arduino tracker will send back the data to the PC to be displayed on the interface. 


\section{Result and Analysis}

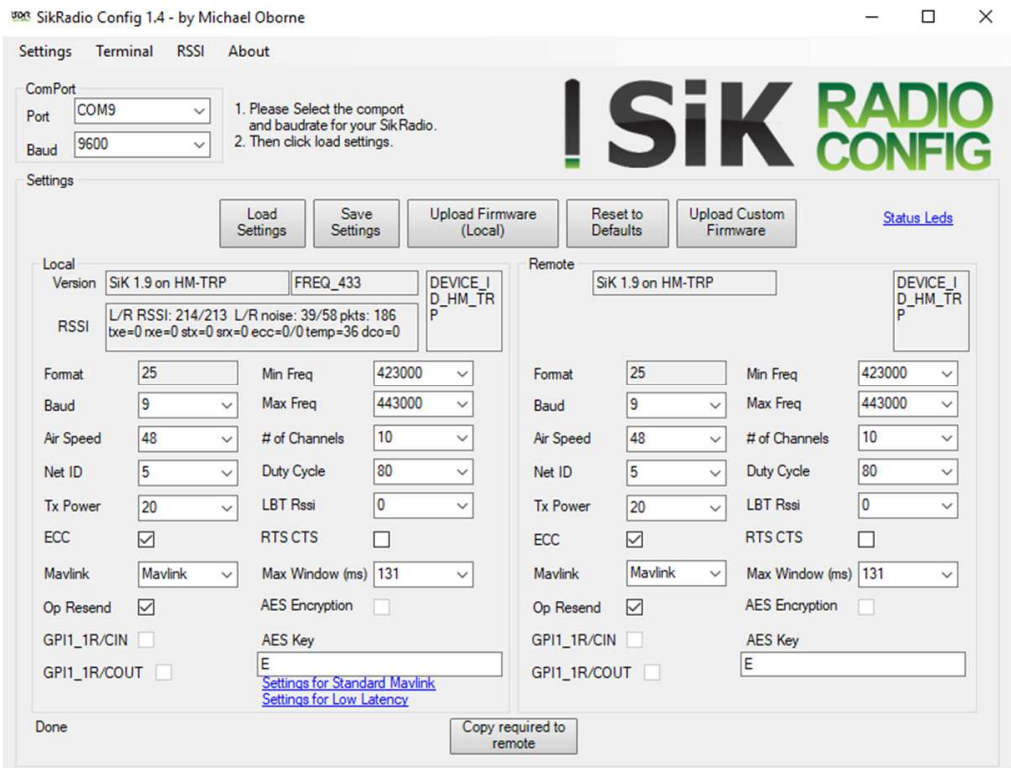

Figure 2. Radio module setting

Figure 2 shows an application to configure the radio frequency in order to synchronize the air module with the ground module so that the air module can send data from the payload to the ground module to be displayed on the interface. This configuration requires two PCs and two RF modules.

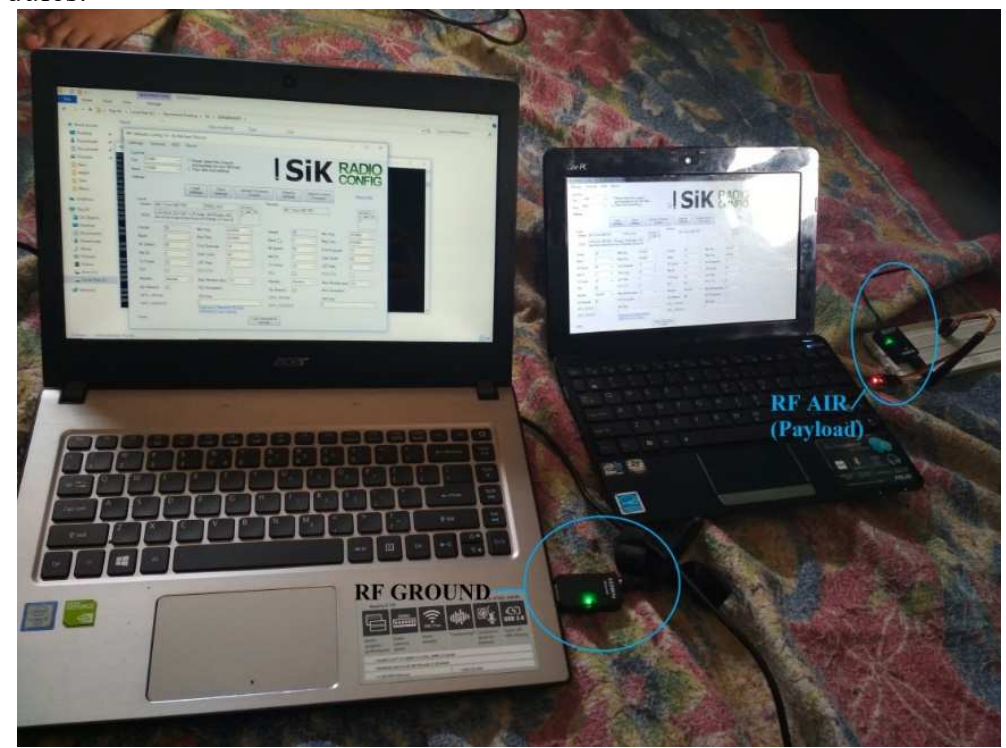

Figure 3. Connecting Ground RF Module to Air RF Module 
Figure 3 illustrates the connection between the air RF module and the ground RF module. The connection is indicated by the constant light (not blinking).

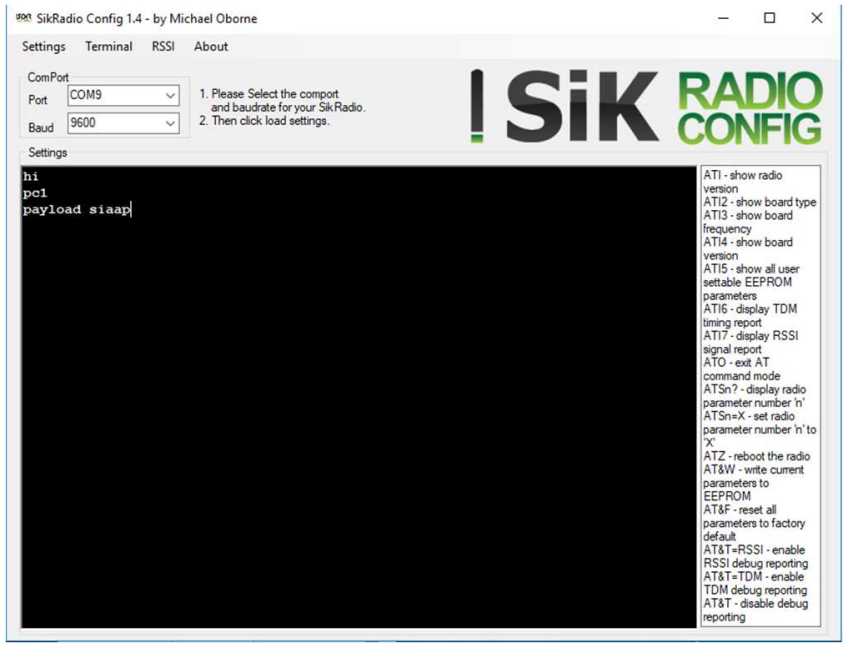

Figure 4. Communication Testing between Transmitter and Receiver Module

Figure 4 presents the communication testing between transmitter and receiver module. It shows that the two modules are successfully connected, indicating that the RF $433 \mathrm{Mhz}$ is ready to use.

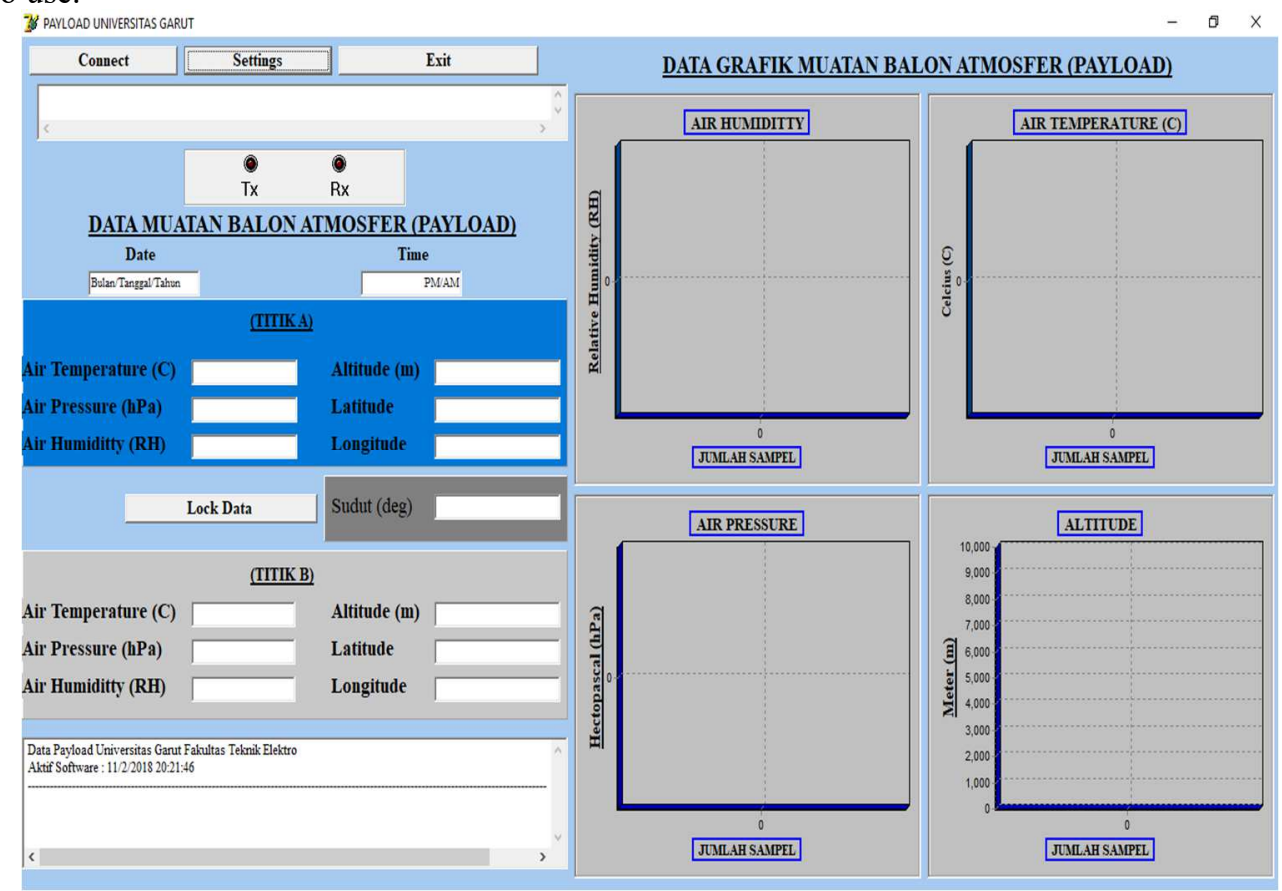

Figure 5. Delphi User Interface 
Figure 5 shows the interface on which data from the payload can be displayed.

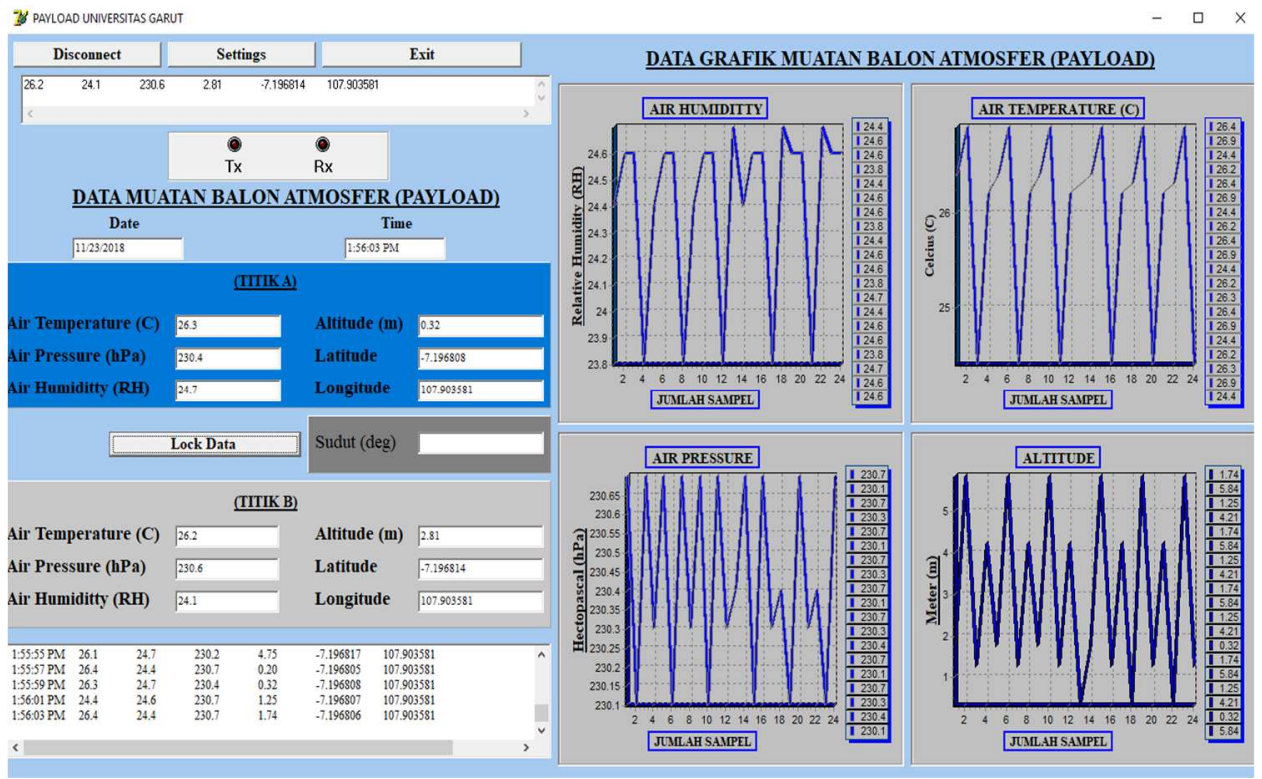

Figure 6. Data as Displayed on the User Interface

Figure 6 shows how data is displayed on the interface. It shows that all component works well.

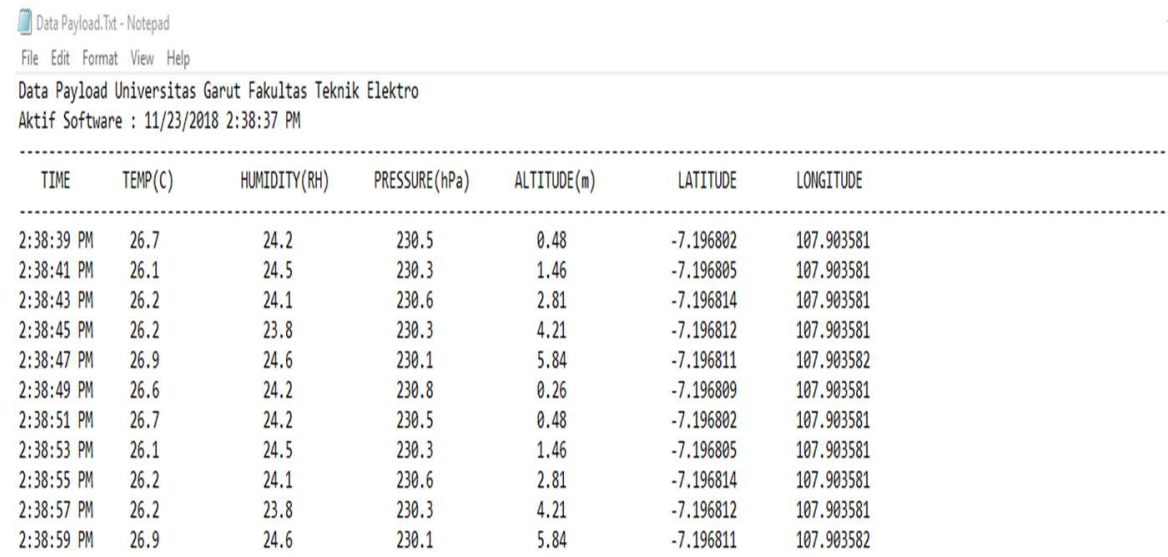

Figure 7. Payload Data History as Displayed on Notepad

Figure 7 shows how data is recorded in the form of *txt file. The users can anytime check this history. This data is stored using a 5 second interval. 
Table 1. Payload Data with Error List

\begin{tabular}{|c|c|c|c|c|c|c|}
\hline TIME & $\begin{array}{c}\text { PRESSUR } \\
\text { E (hPa) }\end{array}$ & $\begin{array}{l}\text { TEMP } \\
\text { (C) }\end{array}$ & $\begin{array}{c}\text { HUMIDIT } \\
\text { Y (RH) }\end{array}$ & LAT & LONG & ALT \\
\hline $\begin{array}{c}: 32: 12 \\
\text { PM }\end{array}$ & 928.10 & 25.94 & 63.20 & $\begin{array}{c}- \\
7.113160\end{array}$ & $\begin{array}{c}107.90270 \\
2\end{array}$ & 745.70 \\
\hline $\begin{array}{c}\text { 4:32:17 } \\
\text { PM }\end{array}$ & 928.05 & 25.94 & 63.10 & $\begin{array}{c}- \\
7.113160\end{array}$ & $\begin{array}{c}107.90270 \\
2\end{array}$ & 745.40 \\
\hline $\begin{array}{c}4: 32: 22 \\
\text { PM }\end{array}$ & 928.12 & 25.94 & 63.20 & $\begin{array}{c}- \\
7.113135\end{array}$ & $\begin{array}{c}107.90271 \\
7\end{array}$ & 745.50 \\
\hline $\begin{array}{c}\text { 4:32:27 } \\
\text { PM }\end{array}$ & 928.11 & 25.81 & 62.40 & $\begin{array}{c}- \\
7.113109\end{array}$ & $\begin{array}{c}107.90278 \\
6\end{array}$ & 742.90 \\
\hline $\begin{array}{c}4: 32: 32 \\
\text { PM }\end{array}$ & 928.15 & 25.75 & 63.10 & $\begin{array}{c}- \\
7.113066\end{array}$ & $\begin{array}{c}107.90283 \\
9\end{array}$ & 743.40 \\
\hline $\begin{array}{c}4: 32: 37 \\
\text { PM }\end{array}$ & 928.09 & 25.75 & 63.50 & $\begin{array}{c}- \\
7.113059\end{array}$ & $\begin{array}{c}107.90284 \\
7\end{array}$ & 743.30 \\
\hline $\begin{array}{c}4: 32: 42 \\
\text { PM }\end{array}$ & 928.13 & 25.81 & 65.30 & $\begin{array}{c}- \\
7.113069\end{array}$ & $\begin{array}{c}107.90284 \\
7\end{array}$ & 743.40 \\
\hline $\begin{array}{c}4: 32: 47 \\
\text { PM }\end{array}$ & 928.12 & 25.81 & 65.00 & $\begin{array}{c}- \\
7.113117\end{array}$ & $\begin{array}{c}107.90283 \\
9\end{array}$ & 742.70 \\
\hline $\begin{array}{c}4: 32: 52 \\
\text { PM }\end{array}$ & 928.12 & 25.75 & 64.60 & $\begin{array}{c}- \\
7.113123\end{array}$ & $\begin{array}{c}107.90284 \\
7\end{array}$ & 743.20 \\
\hline $\begin{array}{c}4: 32: 57 \\
\text { PM }\end{array}$ & 928.12 & 25.81 & 66.30 & $\begin{array}{c}- \\
7.113142\end{array}$ & $\begin{array}{c}107.90283 \\
9\end{array}$ & 743.20 \\
\hline $\begin{array}{c}\text { 4:33:02 } \\
\text { PM }\end{array}$ & 928.17 & 25.81 & 65.20 & $\begin{array}{c}- \\
7.113162\end{array}$ & $\begin{array}{c}107.90286 \\
2\end{array}$ & 743.70 \\
\hline $\begin{array}{c}\text { 4:33:07 } \\
\text { PM }\end{array}$ & 928.10 & 25.75 & 64.50 & $\begin{array}{c}- \\
7.113172\end{array}$ & $\begin{array}{c}107.90285 \\
4\end{array}$ & 743.60 \\
\hline $\begin{array}{c}\text { 4:33:12 } \\
\text { PM }\end{array}$ & 928.15 & 25.81 & 66.70 & $\begin{array}{c}- \\
7.113172\end{array}$ & $\begin{array}{c}107.90285 \\
4\end{array}$ & 743.60 \\
\hline $\begin{array}{c}\text { 4:33:17 } \\
\text { PM }\end{array}$ & 928.17 & 25.87 & 68.20 & $\begin{array}{c}- \\
7.113178\end{array}$ & & \\
\hline $\begin{array}{c}\text { 4:33:22 } \\
\text { PM }\end{array}$ & 107.902839 & 743.00 & & & & \\
\hline $\begin{array}{c}4: 33: 27 \\
\text { PM }\end{array}$ & 928.11 & 25.75 & 65.80 & $\begin{array}{c}- \\
7.113219\end{array}$ & $\begin{array}{c}107.90281 \\
6\end{array}$ & 743.20 \\
\hline $\begin{array}{c}4: 33: 32 \\
\text { PM }\end{array}$ & 928.15 & 25.69 & 66.00 & $\begin{array}{c}- \\
7.113300\end{array}$ & $\begin{array}{c}107.90281 \\
6\end{array}$ & 742.70 \\
\hline $\begin{array}{c}\text { 4:33:37 } \\
\text { PM }\end{array}$ & 928.15 & 25.50 & 65.20 & $\begin{array}{c}- \\
7.113283\end{array}$ & $\begin{array}{c}107.90288 \\
5\end{array}$ & 744.20 \\
\hline $\begin{array}{c}\text { 4:33:42 } \\
\text { PM }\end{array}$ & 928.10 & 25.44 & 64.80 & $\begin{array}{c}- \\
7.113280\end{array}$ & $\begin{array}{c}107.90289 \\
3\end{array}$ & 744.50 \\
\hline $\begin{array}{c}\text { 4:33:47 } \\
\text { PM }\end{array}$ & 928.05 & 25.44 & 65.70 & $\begin{array}{c}- \\
7.113280\end{array}$ & $\begin{array}{c}107.90290 \\
0\end{array}$ & 745.40 \\
\hline $\begin{array}{c}4: 33: 52 \\
\text { PM }\end{array}$ & 928.04 & 25.44 & 65.00 & $\begin{array}{c}- \\
7.113268\end{array}$ & $\begin{array}{c}107.90290 \\
8\end{array}$ & 746.40 \\
\hline $\begin{array}{c}\text { 4:33:57 } \\
\text { PM }\end{array}$ & 928.07 & 25.37 & 65.20 & $\begin{array}{c}- \\
7.113263\end{array}$ & $\begin{array}{c}107.90291 \\
5\end{array}$ & 747.60 \\
\hline
\end{tabular}




\begin{tabular}{|c|c|c|c|c|c|c|}
\hline $\begin{array}{c}\text { 4:34:02 } \\
\text { PM }\end{array}$ & 928.04 & 25.31 & 65.30 & $\begin{array}{c}- \\
7.113254\end{array}$ & $\begin{array}{c}107.90291 \\
5\end{array}$ & 746.90 \\
\hline $\begin{array}{c}\text { 4:34:07 } \\
\text { PM }\end{array}$ & 928.06 & 25.25 & 64.90 & $\begin{array}{c}- \\
7.113225\end{array}$ & $\begin{array}{c}107.90293 \\
8\end{array}$ & 747.10 \\
\hline $\begin{array}{c}4: 34: 12 \\
\text { PM }\end{array}$ & 928.05 & 25.25 & 66.10 & $\begin{array}{c}- \\
7.113220\end{array}$ & $\begin{array}{c}107.90295 \\
4\end{array}$ & 748.20 \\
\hline $\begin{array}{c}4: 34: 17 \\
\text { PM }\end{array}$ & 928.04 & 25.25 & 67.60 & $\begin{array}{c}- \\
7.113219\end{array}$ & $\begin{array}{c}107.90295 \\
4\end{array}$ & 748.40 \\
\hline $\begin{array}{c}4: 34: 22 \\
\text { PM }\end{array}$ & 928.05 & 25.25 & 66.20 & $\begin{array}{c}- \\
7.113293\end{array}$ & $\begin{array}{c}107.90295 \\
4\end{array}$ & 748.10 \\
\hline $\begin{array}{c}4: 34: 27 \\
\text { PM }\end{array}$ & 928.09 & 25.19 & 66.00 & $\begin{array}{c}- \\
7.113324\end{array}$ & $\begin{array}{c}107.90297 \\
6\end{array}$ & 749.70 \\
\hline $\begin{array}{c}4: 34: 32 \\
\text { PM }\end{array}$ & 928.08 & 25.19 & & & & \\
\hline $\begin{array}{c}\text { 4:34:37 } \\
\text { PM }\end{array}$ & 66.20 & $\begin{array}{c}- \\
7.11332 \\
8\end{array}$ & 107.902984 & 750.50 & & \\
\hline $\begin{array}{c}4: 34: 42 \\
\text { PM }\end{array}$ & 928.07 & 25.12 & 65.60 & $\begin{array}{c}- \\
7.113327\end{array}$ & $\begin{array}{c}107.90298 \\
4\end{array}$ & 751.30 \\
\hline $\begin{array}{c}4: 34: 47 \\
\text { PM }\end{array}$ & 928.07 & 25.12 & 66.20 & $\begin{array}{c}- \\
7.113326\end{array}$ & $\begin{array}{c}107.90298 \\
4\end{array}$ & 751.90 \\
\hline $\begin{array}{c}4: 34: 52 \\
\text { PM }\end{array}$ & 928.08 & 25.12 & 66.40 & $\begin{array}{c}- \\
7.113323\end{array}$ & $\begin{array}{c}107.90298 \\
4\end{array}$ & 753.00 \\
\hline $\begin{array}{c}4: 34: 57 \\
\text { PM }\end{array}$ & 928.07 & 25.12 & 67.10 & $\begin{array}{c}- \\
7.113326\end{array}$ & $\begin{array}{c}107.90299 \\
2\end{array}$ & 752.80 \\
\hline $\begin{array}{c}\text { 4:35:02 } \\
\text { PM }\end{array}$ & 928.09 & 25.19 & 69.40 & $\begin{array}{c}- \\
7.113328\end{array}$ & $\begin{array}{c}107.90298 \\
4\end{array}$ & 752.80 \\
\hline $\begin{array}{c}\text { 4:35:07 } \\
\text { PM }\end{array}$ & 928.08 & 25.19 & 69.50 & $\begin{array}{c}- \\
7.113332\end{array}$ & $\begin{array}{c}107.90293 \\
8\end{array}$ & 751.90 \\
\hline $\begin{array}{c}4: 35: 12 \\
\text { PM }\end{array}$ & 928.17 & 25.12 & 68.50 & $\begin{array}{c}- \\
7.113302\end{array}$ & $\begin{array}{c}107.90289 \\
3\end{array}$ & 750.60 \\
\hline $\begin{array}{c}\text { 4:35:17 } \\
\text { PM }\end{array}$ & 928.12 & 25.06 & 67.70 & $\begin{array}{c}- \\
7.113294\end{array}$ & $\begin{array}{c}107.90285 \\
4\end{array}$ & 749.80 \\
\hline $\begin{array}{c}4: 35: 22 \\
\text { PM }\end{array}$ & 928.15 & 25.06 & 66.90 & $\begin{array}{c}- \\
7.113298\end{array}$ & $\begin{array}{c}107.90285 \\
4\end{array}$ & 748.60 \\
\hline $\begin{array}{c}4: 35: 27 \\
\text { PM }\end{array}$ & 928.17 & 25.06 & 67.50 & $\begin{array}{c}- \\
7.113300\end{array}$ & $\begin{array}{c}107.90284 \\
7\end{array}$ & 747.40 \\
\hline $\begin{array}{c}4: 35: 32 \\
\text { PM }\end{array}$ & 928.15 & 25.00 & 67.80 & $\begin{array}{c}- \\
7.113302\end{array}$ & $\begin{array}{c}107.90283 \\
9\end{array}$ & 746.80 \\
\hline $\begin{array}{c}4: 35: 37 \\
\text { PM }\end{array}$ & 928.15 & 25.00 & 66.70 & $\begin{array}{c}- \\
7.113301\end{array}$ & $\begin{array}{c}107.90283 \\
9\end{array}$ & 747.20 \\
\hline $\begin{array}{c}4: 35: 42 \\
\text { PM }\end{array}$ & 928.17 & 25.00 & 67.00 & $\begin{array}{c}- \\
7.113302\end{array}$ & $\begin{array}{c}107.90283 \\
9\end{array}$ & 747.30 \\
\hline \multicolumn{7}{|l|}{$\begin{array}{c}\text { 4:35:47 } \\
\text { PM }\end{array}$} \\
\hline $\begin{array}{c}\text { 4:35:52 } \\
\text { PM }\end{array}$ & 928.16 & 25.00 & 66.90 & $\begin{array}{c}- \\
7.113304\end{array}$ & $\begin{array}{c}107.90284 \\
7\end{array}$ & 746.80 \\
\hline
\end{tabular}




\begin{tabular}{|c|c|c|c|c|c|c|}
\hline $\begin{array}{c}4: 35: 57 \\
\text { PM }\end{array}$ & 928.22 & 25.00 & 67.40 & $\begin{array}{c}- \\
7.113292\end{array}$ & $\begin{array}{c}107.90281 \\
6\end{array}$ & 746.10 \\
\hline $\begin{array}{c}4: 36: 02 \\
\text { PM }\end{array}$ & 928.30 & 25.00 & 67.20 & $\begin{array}{c}- \\
7.113241\end{array}$ & $\begin{array}{c}107.90282 \\
4\end{array}$ & 745.90 \\
\hline $\begin{array}{c}\text { 4:36:07 } \\
\text { PM }\end{array}$ & 928.24 & 24.94 & 67.00 & $\begin{array}{c}- \\
7.113196\end{array}$ & $\begin{array}{c}107.90283 \\
2\end{array}$ & 745.50 \\
\hline $\begin{array}{c}4: 36: 12 \\
\text { PM }\end{array}$ & 928.28 & 24.94 & 67.10 & $\begin{array}{c}- \\
7.113191\end{array}$ & $\begin{array}{c}107.90283 \\
2\end{array}$ & 745.70 \\
\hline $\begin{array}{c}\text { 4:36:17 } \\
\text { PM }\end{array}$ & 928.30 & 24.94 & 66.90 & $\begin{array}{c}- \\
7.113174\end{array}$ & $\begin{array}{c}107.90278 \\
6\end{array}$ & 745.90 \\
\hline $\begin{array}{c}4: 36: 22 \\
\text { PM }\end{array}$ & 928.31 & 24.94 & 66.50 & $\begin{array}{c}- \\
7.113148\end{array}$ & $\begin{array}{c}107.90273 \\
2\end{array}$ & 747.10 \\
\hline $\begin{array}{c}4: 36: 27 \\
\text { PM }\end{array}$ & 928.24 & 24.94 & 67.00 & $\begin{array}{c}- \\
7.113149\end{array}$ & $\begin{array}{c}107.90272 \\
5\end{array}$ & 747.50 \\
\hline $\begin{array}{c}4: 36: 32 \\
\text { PM }\end{array}$ & 928.18 & 24.94 & 66.60 & $\begin{array}{c}- \\
7.113149\end{array}$ & $\begin{array}{c}107.90271 \\
7\end{array}$ & 748.10 \\
\hline $\begin{array}{c}4: 36: 37 \\
\text { PM }\end{array}$ & 928.21 & 24.94 & 67.20 & $\begin{array}{c}- \\
7.113149\end{array}$ & $\begin{array}{c}107.90271 \\
7\end{array}$ & 747.90 \\
\hline $\begin{array}{c}4: 36: 42 \\
\text { PM }\end{array}$ & 928.19 & 24.94 & 67.60 & $\begin{array}{c}- \\
7.113150\end{array}$ & $\begin{array}{c}107.90270 \\
9\end{array}$ & 747.50 \\
\hline
\end{tabular}

Table 1 shows that the data is recorded in a 5 second interval. The yellow parts show that some data are missing. The amount data passing through a system from input to output or throughput can be calculated using the following formula:

$$
\text { Throughput }=\frac{\text { Amount of transmitted data }}{\text { Transmission interval time }} .
$$

The amount of transmitted data is calculated using the following formula:

$$
\text { Data character range } \times \text { user bits }
$$

Table 1 shows that the data has 79 characters; each character has 1 byte or 8 bits. Therefore, the amount of transmitted data is 15.8 bytes $/ \mathrm{s}$ :

$$
\text { Throughput }=\frac{79}{5}=15.8 \text { bytes } / \text { second }
$$

To fix this error, we call the data again in another time. The error still can not to fix the data at the same time when the error appear.

\section{Conclusion}

The designed interface managed to display information received from a payload in the form of numerical and graphical data. These data are stored in a 5 second interval in the form of *txt file format. Sometimes, errors occur during data recording. The throughput is 15.8 bytes per second, and the number of transmitted characters is 79 , meaning that the transmitted data is 632 bits or 79 bytes. 


\section{Acknowledgement}

This publication is made possible by the support of Universitas Garut, Indonesia

\section{References}

[1] " "Homepage | Komurindo-Kombat 2018." [Online]. Available: http://komurindokombat.lapan.go.id/. [Accessed: 15-Jan-2020].

[2] "150.000 Ground Segment Satelit Satria Ditender Terpisah - Teknologi Bisnis.com." [Online]. Available: https://teknologi.bisnis.com/read/20190613/101/933430/150.000ground-segment-satelit-satria-ditender-terpisah. [Accessed: 15-Jan-2020].

[3] H. Junianto, "Sistem Komunikasi Satelit." .

[4] H. Liu, H. Dai, S. Wu, and S. Huang, "Chord-based Resource Identifier-to-Locator Mapping and Searching for the Future Internet," TELKOMNIKA (Telecommunication Comput. Electron. Control., vol. 12, no. 3, p. 703, Sep. 2014, doi: 10.12928/v12i3.99.

[5] "Modern Telemetry - Google Buku." [Online]. Available: https://books.google.co.id/books?id=7XSfDwAAQBAJ\&pg=PR2\&lpg=PR2\&dq=Kre jcar, + O. + Modern + Telemetry. + Rijeka,Croatia:+Intech.+. $+(2011) . \&$ source=bl\&ots=R5 nMMyVlBf\&sig=ACfU3U0DdA8PBjApnjN46BzZIC--

91gBnA\&hl=id\&sa=X\&ved=2ahUKEwi9mLitl4XnAhUPA3IKHf7eC0oQ6AEwAXo ECAoQAQ $\# \mathrm{v}=$ onepage $\& \mathrm{q}=$ Krejcar $\% 2 \mathrm{C} \mathrm{O}$. Modern Telemetry.

Rijeka\%2CCroatia\%3A Intech. . (2011).\&f=false. [Accessed: 15-Jan-2020].

[6] A. Devarajan et al., "Electronic instrumentation capabilities for scientific ballooning in India," cosp, vol. 42, p. PSB.1-45-18, 2018.

[7] "-Length, weight, and tagging data for small- mouth bass implanted with... | Download Table." [Online]. Available: https://www.researchgate.net/figure/Length-weight-andtagging-data-for-small-mouth-bass-implanted-with-radio_tbl1_254310689. [Accessed: 15-Jan-2020].

[8] “CX-D80 DAI NIPPON, CX-D80H DAI NIPPON, CX 330 DAI NIPPON - FUORI PRODUZIONE, Ermes - Distribuzione di stampanti termografiche per carte plastiche." [Online]. Available: http://www.ermes-online.it/cgi$\mathrm{bin} /$ pub/wa_public.cgi? _mode=list_prod\&cat_level $=2 \&$ cat2_id $=3 \& \mathrm{gclid}=\mathrm{Cj} 0 \mathrm{KCQi}$ AjfvwBRCkARIsAIqSWlOnYqZlwtfND9MBSQuVXxwq6J6BN6wqijxu9Y7rZYXro hvRimqbQ1MaAqX9EALw_wcB. [Accessed: 15-Jan-2020].

[9] "ESC Training Grants." [Online]. Available: https://www.escardio.org/Education/Career-Development/Grants-andfellowships/ESC-traininggrants?gclid=Cj0KCQiAjfvwBRCkARIsAIqSWlPkvfJK8KFYeigLDaEHefzTqy4EO XSC9AjYdSZ4EbMShZLtyasOs2MaAi4yEALw_wcB. [Accessed: 15-Jan-2020].

[10] "Fixed-Wing Air Ambulance - RACQ LifeFlight Rescue." [Online]. Available: https://www.lifeflight.org.au/airambulance/?gclid=Cj0KCQiAjfvwBRCkARIsAIqSWIMQ5YI50OALcd1edhh56pBp- 
i8-k81_7CiWs_pha5o63deJXkzw_JUaAtmREALw_wcB. [Accessed: 15-Jan-2020].

[11] “Trainings \& Workshops - QGLUE.” [Online]. Available: https://www.qglue.com/trainings-

workshops/?utm_source $=$ google \&gclid $=$ Cj0KCQiAjfvwBRCkARIsAIqSWIN1ZP1vi POapSSHQ5v8_5jreyUPD7-OhggMBVSXETQfJOS6VVErx7gaAmlwEALw_wcB. [Accessed: 15-Jan-2020].

[12] "Interactive prototypes made easy - Free plan available - by Pidoco." [Online]. Available:

$\mathrm{https} / / /$ pidoco.com/en/lp/prototype?piwik_campaign=en_P20_prototype\&piwik_kwd= design prototypes. [Accessed: 15-Jan-2020].

[13] “Amazon.com: object oriented programming." [Online]. Available: https://www.amazon.com/s?k=object+oriented+programming\&adgrpid=78912437182 \&gclid=Cj0KCQiAjfvwBRCkARIsAIqSWIN5a6bxutj9DVM1 VTICPrFwVMLRvWa 6QGfWfg2-

XX5m_tjkJT8j5lgaAqwcEALw_wcB\&hvadid=393487771131\&hvdev=c\&hvlocphy= 9072569 \&hvnetw $=\mathrm{g} \& \mathrm{hvpos}=1 \mathrm{t} 1$ \&hvqmt $=\mathrm{b} \& \mathrm{hvrand}=6401957316709008079 \& \mathrm{hvtarg}$ id $=$ kwd-10653816\&hydadcr $=14395 \_$10954176\&tag=hydglogoo-

20\&ref=pd_sl_6c0j3cf2oq_b. [Accessed: 15-Jan-2020].

[14] "Recreation Software - Radically Simplified | RecDesk." [Online]. Available: http://recdesk.com/?gclid=Cj0KCQiAjfvwBRCkARIsAIqSWIODA0J3UBLaE_Cl80f9zp4Eb_Q0YB24ZZoo6tJXIJAEvT-wAzE4eAaAo6tEALw_wcB. [Accessed: 15-Jan-2020].

[15] “Graduate programs.” [Online]. Available: https://www.tue.nl/studeren/graduateschool/graduate-

programs/?gclid=Cj0KCQiAjfvwBRCkARIsAIqSWINi8fckHCCkmBUdqcnZLX2z0g IUvDem63nGL_0cqtJf3_ytjn0mTlwaAhQPEALw_wcB. [Accessed: 15-Jan-2020]. 